Disponível em

http://www.anpad.org.br/rac

RAC, Rio de Janeiro, v. 19, n. 1, art. 6, pp. 107-126, Jan./Fev. 2015

http://dx.doi.org/10.1590/1982-7849rac20151998

$((c))$ EY-NG

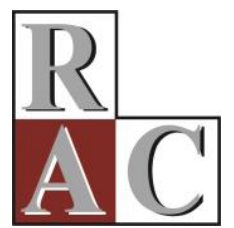

\title{
Motivações da Comunicação Boca a Boca Eletrônica Positiva entre Consumidores no Facebook
}

Motivations for Positive Electronic Word-of-mouth between Consumers on Facebook

Daniel Buarque Tubenchlak Fundação Getulio Vargas - FGV/EBAPE

Diego de Faveri Fundação Getulio Vargas - FGV/EBAPE

Marco Tulio Zanini Fundação Getulio Vargas - FGV/EBAPE

Rafael Goldszmidt Fundação Getulio Vargas - FGV/EBAPE

Artigo recebido em 20.05.2014. Última versão recebida em 29.08.2014. Aprovado em 01.09.2014. 


\title{
Resumo
}

Este estudo buscou investigar as motivações subjacentes ao comportamento de comunicação boca a boca eletrônica (BAB-e) positiva sobre produtos, serviços e marcas no site de redes sociais Facebook, no contexto brasileiro. Para tanto, foram desenvolvidas hipóteses de pesquisa e um modelo teórico para se entender as motivações da comunicação BAB-e positiva nesse contexto. A fim de testar a validade do modelo proposto, um levantamento amostral foi conduzido com 468 usuários do site no Brasil. Os dados foram analisados por meio de modelos de equações estruturais. Os resultados sugerem como motivações significantes a preocupação com outros consumidores e o desejo de interação social, assim como os desejos de extravasar emoções positivas e de ajudar a empresa. A hipótese que previu que as recompensas econômicas afetam positivamente a comunicação BAB-e positiva não foi confirmada, assim, sugerindo que as motivações deste comportamento no Facebook são de natureza social e psicológica mais do que puramente econômica. O teste empírico deu suporte ao papel moderador da força dos laços sociais na relação entre preocupação com outros consumidores e comunicação BAB-e positiva.

Palavras-chave: comunicação boca a boca eletrônica; motivações; redes sociais; sites; Facebook.

\begin{abstract}
The purpose of the present study is to investigate the underlying motivations for positive electronic word of mouth (eWOM) behavior about products, services and brands by Brazilian Facebook users. Research hypotheses and a theoretical model were developed to further our understanding of positive eWOM communication motivations. A survey was conducted with 468 Facebook users in Brazil in order to empirically test the validity of the proposed model. Data was analyzed using structural equations models. Results suggest that concern for other consumers, the desire to engage in social interactions, as well as the desire to share positive emotions and help companies are significant motivations. The hypothesis which predicted that economic rewards are correlated with positive eWOM communication was not confirmed, suggesting that social and psychological motivations are more correlated with eWOM behavior than purely economic reasons for Brazilian Facebook users. The empirical test supported the moderating role of tie strength in the relationship between concern for other consumers and positive eWOM communication.
\end{abstract}

Key words: electronic word-of-mouth communication; motivations; social networks; sites; Facebook. 


\section{Introdução}

O advento da internet ampliou as opções dos consumidores para a busca de informações de outros consumidores e para o compartilhamento de opiniões e conselhos relacionados ao consumo (HennigThurau, Gwinner, Walsh, \& Gremler, 2004; Sweeney, Soutar, \& Mazzarol, 2013). Os consumidores, ao invés das empresas, estão iniciando este diálogo e podem exercer um controle sem precedentes sobre a gestão do conteúdo com o qual eles interagem (Vargo \& Lusch, 2004). Desta forma, deixaram de ser receptores passivos para tornaram-se criadores ativos de informações de consumo. A comunicação boca a boca tradicional (doravante $\mathrm{BAB}$ ), antes, restrita ao pequeno círculo de familiares e amigos, passou a conviver com a comunicação boca a boca eletrônica (doravante BAB-e), um diálogo entre milhares de usuários na internet (H. Lee, Lee, Kim, \& Lee, 2013; Yoo, Sanders, \& Moon, 2013), realizado através de diferentes plataformas: sites de opinião de consumidores (Dellarocas, 2003; Hennig-Thurau et al., 2004), sites de marcas (M. Lee \& Youn, 2009), blogs (Kozinets, Valck, Wojnicki, \& Wilner, 2010) e sites de redes sociais (Chu \& Choi, 2011; Chu \& Kim, 2011; Wolny \& Mueller, 2013). Neste cenário, destaca-se o surgimento dos sites de redes sociais na internet, como o Facebook, LinkedIn e Twitter. Desde sua introdução, na segunda metade da década de 1990, estes sites têm atraído milhões de usuários em todo o mundo (Boyd \& Ellison, 2007).

\section{Boyd e Ellinson (2007, p. 211) definem sites de redes sociais como}

serviços baseados na web que permitem aos indivíduos: (1) construir um perfil público ou semipúblico dentro de um sistema delimitado; (2) articular uma lista de outros usuários com quem eles compartilham uma conexão; e (3) ver e percorrer a sua lista de conexões e aquelas feitas por outras pessoas dentro do sistema.

Lançado em 2004, atualmente, o Facebook é o maior site de redes sociais do mundo, contando com 1,06 bilhão de usuários ativos no final de 2012. Neste mesmo período, alcançou a marca de 67 milhões de usuários ativos no Brasil, ocupando a posição de liderança entre os sites de redes sociais no país (Facebook, 2012). Por meio da participação no Facebook, os consumidores acessam e utilizam recursos incorporados ao site, tais como informações e ideias, os quais facilitam as interações sociais e, consequentemente, a disseminação da comunicação BAB-e sobre produtos, serviços e marcas nesse ambiente virtual.

Apesar da importância da comunicação BAB-e na disciplina de marketing e do aumento de interesse pelo tema, o estudo de suas motivações tem recebido atenção limitada até a presente data. Os poucos estudos realizados consideraram países distintos do Brasil e abordaram diferentes plataformas, sendo que foram raras as pesquisas que contemplaram especificamente o contexto dos sites de redes sociais, como o Facebook e Twitter (Wolny \& Mueller, 2013).

A heterogeneidade do BAB-e entre diferentes plataformas é exemplificada por uma pesquisa sobre experiência de compra e consumo de serviços de viagens de férias na Holanda, realizada por Bronner e Hoog (2010). Os resultados sugerem que as motivações da comunicação BAB-e diferem entre os distintos tipos de sites da internet. Com base nos resultados da pesquisa, os autores propuseram uma tipologia baseada em motivações e identificaram dois segmentos distintos: (a) turistas com motivações mais autodirigidas (como, por exemplo, recompensas econômicas), que preferem postar seus comentários em sites das próprias empresas de turismo, em que suas motivações de natureza egoísta podem ser mais recompensadas; (b) turistas com motivações mais dirigidas para os outros (como, por exemplo, ajudar outros turistas em suas decisões de compra), que preferem postar em sites com conteúdo gerado pelos próprios consumidores, pois parecem valorizar o diálogo e o suporte encontrado nesse ambiente.

Apesar de ser um fenômeno global, pesquisa de Chu e Kim (2011) sugere que o comportamento de BAB-e em sites de redes sociais é culturalmente moldado, e pode variar de país para país em função de diferenças culturais. Os autores identificaram que os consumidores chineses são mais ativos em comunicação BAB-e em sites de redes sociais, confiam mais em seus contatos e apresentam laços sociais 
mais fortes do que os consumidores americanos, reflexo de uma orientação cultural mais coletivista e relações sociais mais horizontais na China do que nos Estados Unidos.

Dado a comunicação BAB-e ser impactada pela cultura geográfica (Chu \& Choi, 2011), bem como pelo tipo de site utilizado para sua emissão (Bronner \& Hoog, 2010), este estudo busca preencher uma lacuna ao analisar as motivações subjacentes ao comportamento de comunicação BAB-e positiva sobre produtos, serviços e marcas no site de redes sociais Facebook no contexto brasileiro. Permite, assim, a extensão da teoria para um diferente contexto. Mais além, desenvolve os modelos teóricos anteriores pela inclusão da força dos laços sociais como moderadora da relação entre motivações e BABe, variável esta que havia sido considerada, antes, apenas, em seu efeito direto.

Para tanto, foi desenvolvido um modelo teórico a partir da tipologia de motivações da comunicação BAB-e de Hennig-Thurau, Gwinner, Walsh e Gremler (2004). O construto força dos laços sociais (Granovetter, 1973) foi incluído no modelo, pois apresenta grande relevância para o entendimento da comunicação $\mathrm{BAB}$ tradicional $(\mathrm{BAB})$ e eletrônica (BAB-e), conforme abordado nos estudos de J. J. Brown e Reingen (1987) e Chu e Kim (2011). O modelo foi testado através de um levantamento amostral com 468 usuários do Facebook no Brasil.

\section{Revisão da Literatura e Hipóteses}

\section{Comunicação boca a boca eletrônica (BAB-e)}

Profissionais de diferentes áreas têm reconhecido a importância da comunicação BAB-e por mais de meio século, propondo, por exemplo, que o fenômeno afeta a maioria das decisões de compra (Dichter, 1966; Kozinets et al., 2010). A comunicação BAB pode ter um papel decisivo ao influenciar mais o comportamento do que a propaganda, sendo percebida como mais confiável porque vem de líderes de opinião, membros de família, amigos e outras fontes percebidas como não comerciais e não relacionadas oficialmente às empresas (Kozinets et al., 2010). Em um dos primeiros trabalhos relevantes sobre o tema, Arndt (1967) definiu a comunicação BAB como "uma comunicação oral e pessoal entre um emissor percebido como não comercial e um receptor, tratando de uma marca, um produto ou um serviço oferecido para venda" (p. 190).

Apesar da sua relevância, essas teorias foram construídas num contexto de marketing sem a presença da internet (Dellarocas, 2003; Hennig-Thurau et al., 2004). No final da década de 1990, Buttle (1998) lançou novas perspectivas sobre o entendimento da comunicação BAB. Segundo o autor, na era da internet, as comunidades on-line geram $\mathrm{BAB}$ virtual que não é face a face. A ampliação do conceito da comunicação BAB para o ambiente virtual passou a ser reconhecida por diversos autores (Breazeale, 2009; Chu \& Kim, 2011; Hennig-Thurau et al., 2004) e novas expressões passaram a ser utilizadas para descrever o comportamento, tais como: boca a boca eletrônico (Goyette, Ricard, Bergeron, \& Marticotte, 2010; Hennig-Thurau et al., 2004) e boca a boca on-line (Groeger \& Buttle, 2014). Hennig-Thurau et al. (2004) definem BAB-e como: "qualquer declaração positiva ou negativa, realizada por clientes potenciais, atuais ou antigos, sobre uma empresa ou um produto, que é disponibilizada para uma variedade de pessoas e instituições através da Internet" (p. 39).

Para o propósito deste estudo, somente a comunicação BAB-e positiva é abordada e o seu conceito é ampliado para incluir três atividades específicas do Facebook: curtir, comentar e publicar conteúdo (texto, imagens, fotos e vídeos) sobre produtos, serviços e marcas, em linha com o estudo de Wolny e Mueller (2013).

Diferentes estudos demonstraram o poder da comunicação BAB-e positiva. Por exemplo, seu impacto foi analisado e comprovado na venda on-line de livros (Chevalier \& Mayzlin, 2006), na receita de bilheteria de filmes (Duan, $\mathrm{Gu}, \&$ Whinston, 2008) e na construção de confiança para realizar compras on-line (Awad \& Ragowsky, 2008). Conhecer melhor as motivações subjacentes a esse 
comportamento permitirá desenvolver ações de comunicação e marketing mais efetivas, que estimulem a propagação da comunicação $\mathrm{BAB}$-e positiva de produtos, serviços e marcas entre os consumidores brasileiros no Facebook.

\section{Motivações para fornecimento da comunicação boca a boca eletrônica (BAB-e)}

Uma quantidade limitada de pesquisas sobre as motivações da comunicação BAB-e foi publicada até a presente data. Neste trabalho, adota-se a tipologia de motivações para a emissão da comunicação BAB-e fornecida por Hennig-Thurau et al. (2004), desenvolvida a partir dos estudos da comunicação BAB tradicional de Dichter (1966), Engel, Blackwell e Miniard (1993) e Sundaram, Mitra e Webster (1998), bem como dos estudos sobre comunidades virtuais de Balasubramanian e Mahajan (2001). O estudo de Hennig-Thurau et al. (2004) foi validado empiricamente em uma amostra de usuários alemães de plataformas de opinião de consumidores e identificou oito fatores motivacionais, sendo que seis deles explicam a comunicação BAB-e positiva, foco do presente estudo: preocupação com os outros consumidores, desejo de ajudar a empresa, benefício social, autoelevação, recompensas econômicas e extravasar emoções positivas.

A preocupação com os outros consumidores pode ser definida como o desejo de ajudá-los com suas decisões de compra, sem antecipar alguma recompensa em troca (Hennig-Thurau et al., 2004; Sundaram, Mitra, \& Webster, 1998). Neste caso, o que prevalece é a intenção de ajudar e de expressar sentimentos de cuidado, amizade e carinho (Dichter, 1966). Para Hennig-Thurau et al. (2004), esta preocupação com outros consumidores está intimamente relacionada com o conceito do altruísmo, conforme abordado na literatura de marketing, por Price, Feick e Guskey (1995). Um dos aspectos relevantes da recomendação espontânea é justamente esta preocupação natural de ajudar a outra parte com que se mantém algum tipo de relação (Sundaram et al., 1998). No contexto do Facebook, os usuários tendem a usar identidades verdadeiras e a estabelecer relacionamentos com contatos que já são parte de sua rede social de relacionamento, como, por exemplo, colegas de trabalho, amigos e família, caracterizados por relações pessoais mais próximas e amigáveis. Portanto, espera-se que uma maior preocupação em ajudar a rede de contatos em suas decisões de compra ou consumo impacte de forma positiva a comunicação BAB-e positiva. Nesse sentido, a seguinte hipótese é proposta:

H1: A Preocupação com outros consumidores (POC) está positivamente associada à comunicação BAB-e positiva no Facebook.

O desejo de ajudar a empresa, primeiramente, identificado no estudo de Sundaram et al. (1998) e, posteriormente, na pesquisa de Hennig-Thurau et al. (2004), no contexto da internet, baseia-se no mesmo fundo psicológico da motivação de ajudar os outros consumidores e está relacionado com a motivação geral de altruísmo. Pode ser definido como o resultado da satisfação do consumidor e o seu subsequente desejo de dar algo em troca ao ajudar a empresa (Hennig-Thurau et al., 2004; Sundaram et al., 1998). O efeito pretendido do BAB-e do consumidor é tornar ou manter a empresa bem-sucedida (Hennig-Thurau et al., 2004). Segundo a teoria da desconfirmação de expectativas, proposta por Oliver (1980), os consumidores, através de um processo cognitivo, avaliam o desempenho de um produto ou serviço e comparam essas avaliações com suas expectativas anteriores ao consumo ou à compra. $\mathrm{Na}$ medida em que o desempenho do produto ou serviço excede as expectativas do consumidor, ocorre a desconfirmação positiva, e o mesmo tende a um estado de encanto, o que motiva este consumidor a compartilhar com outros, sua experiência positiva. Diversos estudos comprovaram o efeito significativo da satisfação no comportamento de BAB (Swan \& Oliver, 1989; T. J. Brown, Barry, Dacin, \& Gunst, 2005). Para Hennig-Thurau et al. (2004), este motivo também pode ser explicado pela teoria da equidade (Swan \& Oliver, 1989), que sugere que os indivíduos desejam trocas equitativas e justas. Neste caso, a recomendação é uma forma de restaurar o equilíbrio do consumidor, dessa maneira, ocasionado pelo sentimento de que foi mais beneficiado do que a empresa numa relação de troca. O Facebook facilitou a habilidade dos consumidores de identificar marcas e empresas de interesse e de conectar-se com elas. Por exemplo, através das páginas de fãs, o consumidor pode interagir com organizações e empresas que admira. É uma forma fácil de manifestar publicamente para a rede de relacionamentos que gosta de determinada marca ou empresa. Assim, um maior desejo em ajudar uma determinada empresa 
impactaria de forma positiva a comunicação BAB-e sobre a mesma no Facebook. Com base nessas considerações, a seguinte hipótese é proposta:

H2: O Desejo de ajudar a empresa (DAE) está positivamente associado à comunicação BAB-e positiva no Facebook.

Benefícios sociais como motivação subjacente à comunicação BAB-e foram identificados no estudo de Hennig-Thurau et al. (2004) e podem ser definidos como o desejo de identificação, pertencimento e integração social com a comunidade. Segundo os autores, uma característica da comunicação BAB-e é que os consumidores tornam-se parte de uma comunidade virtual através de suas articulações. Para Kozinets, Valck, Wojnicki e Wilner (2010), a comunicação BAB-e possui um valor de conexão, pois pode facilitar a integração e afiliação de um novo membro numa comunidade virtual. Compartilhar histórias positivas sobre produtos ou marcas no Facebook pode ser uma forma de ter algo a dizer e divertimento, assim, melhorando a atmosfera da comunidade e facilitando o estabelecimento de novos contatos ou a manutenção de contatos antigos com amigos ou conhecidos. Por considerar-se que o principal propósito do Facebook é justamente permitir a socialização entre amigos, colegas e outras pessoas, espera-se que o desejo por interações sociais esteja positivamente associado com a comunicação BAB-e positiva através do site de rede social. Portanto propõe-se que:

H3: O benefício social (BS) está positivamente associado à comunicação BAB-e positiva no Facebook.

A motivação por autoelevação pode ser definida como o desejo de reconhecimento positivo pelos outros, de ser percebido como um comprador inteligente ou um especialista em consumo (HennigThurau et al., 2004; Sundaram et al., 1998). Conforme Sundaram et al. (1998), através do BAB de produtos e marcas, o consumidor busca demonstrar conhecimento, projetar uma imagem de profundo conhecedor num assunto específico e elevar o status. No contexto do Facebook, pode representar o desejo de ser percebido pela sua rede de contatos como um consumidor inteligente, pioneiro, de gosto refinado ou especialista em determinado produto ou serviço. Através de funcionalidades específicas da rede social, como o botão Curtir, o usuário que publica um conteúdo sobre produtos ou marcas pode receber feedback positivo de seus amigos e colegas, o qual fica visível para toda a sua rede de contatos. Uma quantidade elevada de Curtidas (feedbacks positivos) concede ao usuário um nível de exposição e status que pode ser relevante para o seu autoconceito. Assim, a seguinte hipótese é proposta:

H4: A Autoelevação (AE) está positivamente associada à comunicação BAB-e positiva no Facebook.

Conforme validado empiricamente no estudo de Hennig-Thurau et al. (2004), a comunicação BAB-e também pode ser motivada por recompensas econômicas. Segundo os autores, esta motivação pode ser definida como o desejo de receber recompensas econômicas ou financeiras das empresas. Existe uma vasta discussão sobre campanhas de marketing boca a boca envolvendo recompensas econômicas, incluindo suas regras e implicações éticas (Grayson, 2007; Kozinets et al., 2010; Pereira, 2010). Para ganhar recompensas econômicas (por exemplo, descontos e ingressos grátis), no contexto do Facebook, os consumidores podem participar de campanhas, promoções e concursos culturais através da divulgação e compartilhamento de informações sobre produtos e marcas para sua rede de contatos. Logo, espera-se que o desejo por recompensas econômicas esteja positivamente associado com a comunicação BAB-e através do site de rede social. Com base nessas considerações, a seguinte hipótese pode ser enunciada:

H5: A Recompensa Econômica (RE) está positivamente associada à comunicação BAB-e positiva no Facebook.

Segundo Dichter (1966), experiências positivas de compra ou consumo contribuem para o surgimento de uma tensão psicológica no consumidor, ocasionada por um forte desejo de querer compartilhar com alguém a alegria proporcionada pela experiência. Ao analisar a comunicação BAB-e, Hennig-Thurau et al. (2004) sugerem que esta tensão pode ser reduzida ao escrever comentários em plataformas de opinião de consumo virtuais, um comportamento que permite ao consumidor 
compartilhar a alegria da experiência com muitos outros. O Facebook, principalmente a partir de sua consolidação como uma plataforma de acesso móvel através de celulares e tablets, possibilita compartilhar emoções de compra e consumo de forma fácil e rápida com uma rede ampla de contatos. Por exemplo, o usuário pode compartilhar, em tempo real, com sua lista de amigos, um livro que está lendo, um show ao vivo que está assistindo, uma refeição num restaurante ou uma nova música de sua banda favorita. Assim, espera-se que o desejo de extravasar as emoções positivas esteja positivamente associado com a comunicação BAB-e positiva através do Facebook. Logo, a seguinte hipótese é proposta:

H6: Extravasar emoções positivas (EEP) está positivamente associado à comunicação BAB-e positiva no Facebook.

Com o intuito de ampliar o entendimento e melhorar a explicação do comportamento da comunicação BAB-e positiva no Facebook, foi incluído no modelo de motivações, desenvolvido por Hennig-Thurau et al. (2004), o construto força percebida dos laços sociais. Este construto é definido como a intensidade do relacionamento social entre o emissor e o receptor. A força dos laços sociais tem sido abordada em diversos estudos na literatura de marketing e de comportamento do consumidor (Chu \& Kim, 2011; J. J. Brown \& Reingen, 1987). No contexto da internet, o estudo desenvolvido por Chu e Kim (2011) identificou uma associação positiva entre a força dos laços sociais e a comunicação BAB-e em sites de redes sociais. De acordo com os autores, os laços fortes são relativos àqueles amigos com os quais o indivíduo se comunica com frequência e que são percebidos como importantes e próximos, ou seja, são aqueles marcados pela intimidade, proximidade e intencionalidade em criar e manter uma conexão. É razoável, portanto, esperar que uma maior percepção da força dos laços sociais que compõem a rede de contatos no Facebook impacte de forma direta e positiva a comunicação BAB-e no site. Também se espera que a força percebida desses laços sociais modere a relação entre a preocupação com outros consumidores e a comunicação BAB-e. Desta forma, quanto mais forte o laço social entre os membros da rede social, maior seria o efeito da preocupação com outros consumidores na comunicação BAB-e. Nesse sentido, as seguintes hipóteses são propostas:

H7a: A força percebida dos laços sociais (FLS) está positivamente associada à comunicação BAB-e positiva no Facebook.

H7b: A força percebida dos laços sociais (FLS) modera a relação entre a preocupação com outros consumidores (POC) e comunicação BAB-e positiva no Facebook.

Baseando-se na fundamentação teórica e nas hipóteses estabelecidas, a Figura 1 apresenta o modelo teórico a ser investigado.

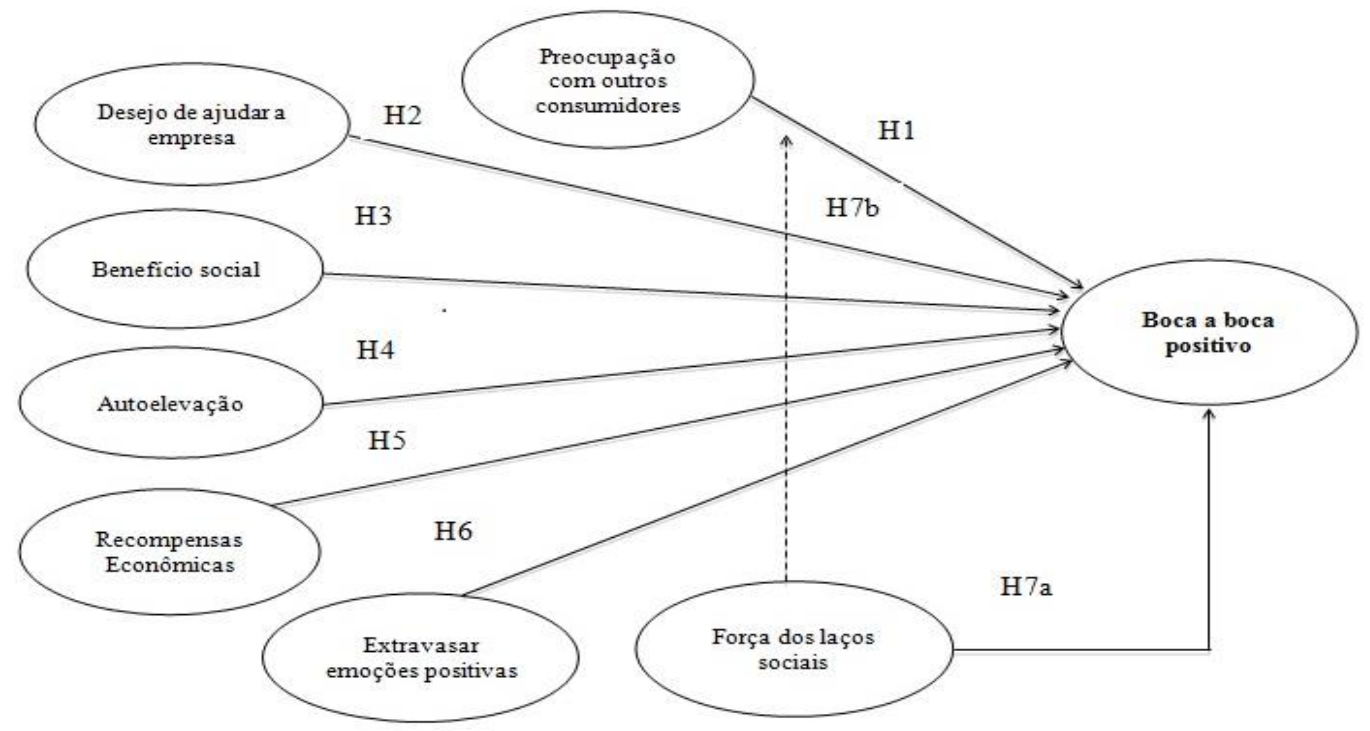

Figura 1. Modelo Teórico

RAC, Rio de Janeiro, v. 19, n. 1, art. 6, pp. 107-126, Jan./Fev. 2015

WWW.anpad.org.br/rac (oc) EY-NC 


\section{Metodologia}

Foi realizada uma pesquisa de corte transversal com coleta de dados por meio de um levantamento amostral (survey) (Botelho \& Almeida, 2009; Fowler, 2014), juntando-se à metodologia adotada em grande parte dos estudos sobre comportamento boca a boca na literatura de marketing. Este desenho de pesquisa permite mensurar comportamentos dos indivíduos no seu contexto real, o que reforça sua validade externa. Porém, embora surveys possibilitem correlacionar os construtos mensurados, também, apresentam limitações quanto à possibilidade de inferência causal, visto que não permitem excluir a existência de problemas de endogeneidade causados por razões como variáveis omitidas (Antonakis, Bendahan, Jacquart, \& Lalive, 2010) ou variância comum ao método (common method variance) (Podsakoff, Mackenzie, \& Podsakoff, 2012). Esses problemas foram parcialmente mitigados no presente estudo pela inclusão de variáveis de controle que poderiam representar explicações alternativas à relação proposta entre os construtos e pela análise de um fator de Harman para verificar a intensidade da variância comum ao método (Podsakoff \& Organ, 1986).

\section{Coleta de dados}

O convite que continha o link do questionário on-line foi enviado por e-mail para alunos e exalunos de MBA da Fundação Getulio Vargas. A pesquisa também foi divulgada nos sites de redes sociais Facebook e LinkedIn. O questionário ficou disponível entre novembro e dezembro de 2012. É importante destacar que esta amostragem, não probabilística, limita a validade externa dos resultados.

\section{Medidas}

A pesquisa bibliográfica possibilitou a identificação de escalas já validadas em estudos anteriores, necessárias à medição dos construtos de interesse do estudo. Primeiramente, os itens das escalas de comunicação BAB-e de Sun, Youn, Wu e Kuntaraporn (2006), de motivações de Hennig-Thurau et al. (2004) e de força percebida dos laços sociais de Chu e Kim (2011) foram traduzidos do inglês para o português por um tradutor brasileiro especializado. Em seguida, um professor de inglês realizou a tradução reversa. As diferenças entre as duas versões foram discutidas com o professor de inglês e três especialistas em marketing para minimizar quaisquer diferenças importantes de significado. A seguir, os itens foram adaptados para o contexto da pesquisa. Conforme recomendado por Hair, Black, Babin e Anderson (2009), tendo em vista garantir um mínimo de três itens para mensuração de cada construto, quando necessário, novos itens foram criados pelos autores, com base na definição teórica dos construtos, e incorporados às escalas existentes (Tabela 1). Todos os itens foram mensurados por meio de uma escala Likert de cinco pontos.

Tabela 1

\section{Construtos e Itens}

\begin{tabular}{llll}
\hline Construto & Código & Indicador & Fonte \\
\hline $\begin{array}{l}\text { Preocupação } \\
\text { com outros } \\
\text { consumidores }\end{array}$ & POC1 & $\begin{array}{l}\text { Porque eu quero ajudar meus contatos no Facebook com Hennig-Thurau et al. } \\
\text { minhas próprias experiências positivas de compra ou (2004) } \\
\text { consumo. }\end{array}$ \\
& POC2 & $\begin{array}{l}\text { Porque eu quero dar aos meus contatos no Facebook a } \\
\text { oportunidade de comprar os produtos ou serviços certos. } \\
\text { POC3 }\end{array}$ & $\begin{array}{l}\text { Porque eu quero ajudar meus contatos no Facebook com } \\
\text { informações úteis sobre produtos, marcas, serviços ou } \\
\text { empresas.* }\end{array}$ \\
\hline
\end{tabular}


Tabela 1 (continuação)

\begin{tabular}{|c|c|c|c|}
\hline Construto & Código & Indicador & Fonte \\
\hline \multirow{3}{*}{$\begin{array}{l}\text { Desejo de } \\
\text { ajudar a } \\
\text { empresa }\end{array}$} & DAE1 & $\begin{array}{l}\text { Porque na minha opinião boas empresas devem ser apoiadas } \\
\text { desta forma no Facebook. }\end{array}$ & \multirow[t]{3}{*}{$\begin{array}{l}\text { Hennig-Thurau et al. } \\
\text { (2004) }\end{array}$} \\
\hline & DAE2 & $\begin{array}{l}\text { Porque eu estou tão satisfeito com a empresa e seus produtos } \\
\text { ou serviços que quero ajudá-la desta forma a ser bem- } \\
\text { sucedida. }\end{array}$ & \\
\hline & DAE3 & $\begin{array}{l}\text { Porque estou tão satisfeito com a empresa que quero dar algo } \\
\text { em troca para ajudá-la a ter sucesso.* }\end{array}$ & \\
\hline \multirow[t]{4}{*}{ Benefício social } & BS1 & $\begin{array}{l}\text { Porque eu encontro, reencontro e mantenho contato com } \\
\text { pessoas legais no Facebook desta forma. }\end{array}$ & \multirow[t]{4}{*}{$\begin{array}{l}\text { Hennig-Thurau et al. } \\
\text { (2004) }\end{array}$} \\
\hline & $\mathrm{BS} 2$ & $\begin{array}{l}\text { Porque é divertido comunicar-me desta forma com outras } \\
\text { pessoas no Facebook. }\end{array}$ & \\
\hline & $\mathrm{BS} 3$ & $\begin{array}{l}\text { Porque eu acho bom conversar no Facebook sobre produtos, } \\
\text { marcas, serviços ou empresas com pessoas que têm opiniões } \\
\text { parecidas. }\end{array}$ & \\
\hline & BS4 & $\begin{array}{l}\text { Não acho divertido comunicar-me desta forma com outras } \\
\text { pessoas no Facebook.* }\end{array}$ & \\
\hline \multirow[t]{5}{*}{ Autoelevação } & AE1 & $\begin{array}{l}\text { Porque desta forma eu mostro aos meus contatos no } \\
\text { Facebook que eu sou um consumidor inteligente. }\end{array}$ & \multirow[t]{5}{*}{$\begin{array}{l}\text { Hennig-Thurau et al. } \\
\text { (2004) }\end{array}$} \\
\hline & AE2 & $\begin{array}{l}\text { Porque eu me sinto bem quando eu posso contar para os meus } \\
\text { contatos no Facebook minhas compras ou experiências de } \\
\text { consumo de sucesso. }\end{array}$ & \\
\hline & AE3 & $\begin{array}{l}\text { Porque desta forma eu mostro aos meus contatos no } \\
\text { Facebook que eu tenho gosto refinado. }{ }^{*}\end{array}$ & \\
\hline & AE4 & $\begin{array}{l}\text { Porque desta forma eu mostro aos meus contatos no } \\
\text { Facebook que eu sou um consumidor pioneiro.* }\end{array}$ & \\
\hline & AE5 & $\begin{array}{l}\text { Porque desta forma eu mostro aos meus contatos no } \\
\text { Facebook que eu sou um especialista no assunto.* }\end{array}$ & \\
\hline \multirow[t]{4}{*}{$\begin{array}{l}\text { Recompensas } \\
\text { econômicas }\end{array}$} & RE1 & $\begin{array}{l}\text { Porque desta forma eu recebo incentivos da empresa (por } \\
\text { exemplo, ingressos grátis ou produtos). }\end{array}$ & \multirow[t]{4}{*}{$\begin{array}{l}\text { Hennig-Thurau et al. } \\
\text { (2004) }\end{array}$} \\
\hline & RE2 & $\begin{array}{l}\text { Eu não espero receber recompensas econômicas (por } \\
\text { exemplo, descontos) pelo que curto, compartilho ou publico } \\
\text { no Facebook.* }\end{array}$ & \\
\hline & RE3 & Porque desta forma eu concorro a prêmios.* & \\
\hline & RE4 & $\begin{array}{l}\text { Porque desta forma eu recebo uma recompensa econômica } \\
\text { (por exemplo, descontos) pelo que curto, compartilho ou } \\
\text { publico no Facebook. }\end{array}$ & \\
\hline \multirow{3}{*}{$\begin{array}{l}\text { Extravasar } \\
\text { emoções } \\
\text { positivas }\end{array}$} & EEP1 & $\begin{array}{l}\text { Porque desta forma eu posso extravasar minha alegria com } \\
\text { experiências gratificantes de compra ou consumo.* }\end{array}$ & \multirow[t]{3}{*}{$\begin{array}{l}\text { Hennig-Thurau et al. } \\
\text { (2004) }\end{array}$} \\
\hline & EEP2 & $\begin{array}{l}\text { Porque desta forma eu posso expressar minha satisfação com } \\
\text { uma boa compra ou experiência de consumo. }\end{array}$ & \\
\hline & EEP3 & $\begin{array}{l}\text { Porque desta forma eu posso reviver o prazer obtido com } \\
\text { experiências gratificantes de compra ou consumo.* }\end{array}$ & \\
\hline
\end{tabular}




\section{Tabela 1 (continuação)}

\begin{tabular}{|c|c|c|c|}
\hline Construto & Código & Indicador & Fonte \\
\hline \multirow[t]{3}{*}{$\begin{array}{l}\text { Forças dos } \\
\text { laços sociais }\end{array}$} & FLS1 & $\begin{array}{l}\text { Aproximadamente com que frequência você se comunica } \\
\text { com os seus contatos no Facebook? }\end{array}$ & Chu e Kim (2011) \\
\hline & FLS2 & $\begin{array}{l}\text { No geral, o quão importante você considera os seus contatos } \\
\text { do Facebook? }\end{array}$ & \\
\hline & FLS3 & $\begin{array}{l}\text { No geral, quão próximo você se sente para com seus contatos } \\
\text { do Facebook? }\end{array}$ & \\
\hline \multirow[t]{9}{*}{$\begin{array}{l}\text { Boca a boca } \\
\text { eletrônica } \\
\text { positiva }\end{array}$} & $\begin{array}{l}\text { BABeC } \\
\text { UR1 }\end{array}$ & $\begin{array}{l}\text { No Facebook, eu gosto de CURTIR as páginas ou } \\
\text { publicações com conteúdo relevante e/ou interessante sobre } \\
\text { meus produtos, marcas, serviços ou empresas favoritas. }\end{array}$ & Sun et al. (2006) \\
\hline & $\begin{array}{l}\text { BABeC } \\
\text { UR2 }\end{array}$ & $\begin{array}{l}\text { Quando estou extremamente satisfeito com um produto, } \\
\text { marca, serviço ou empresa, eu gosto de CURTIR suas } \\
\text { páginas ou publicações interessantes e/ou relevantes no } \\
\text { Facebook.* }\end{array}$ & \\
\hline & $\begin{array}{l}\text { BABeC } \\
\text { UR3 }\end{array}$ & $\begin{array}{l}\text { Eu gosto de CURTIR os comentários de meus contatos que } \\
\text { contenham informações ou opiniões positivas, relevantes } \\
\text { e/ou interessantes sobre produtos, marcas ou empresas de que } \\
\text { gosto, admiro ou me identifico. }\end{array}$ & \\
\hline & $\begin{array}{l}\text { BABeC } \\
\text { OM1 }\end{array}$ & $\begin{array}{l}\text { No Facebook, eu gosto de COMPARTILHAR as publicações } \\
\text { positivas com conteúdo relevante e/ou interessante sobre } \\
\text { meus produtos, marcas, serviços ou empresas favoritas. }\end{array}$ & \\
\hline & $\begin{array}{l}\text { BABeC } \\
\text { OM2 }\end{array}$ & $\begin{array}{l}\text { Quando recebo de um amigo opiniões ou comentários } \\
\text { positivos, relevantes e/ou interessantes sobre um produto, } \\
\text { marca ou empresa de que gosto ou admiro, eu costumo } \\
\text { COMPARTILHAR com meus outros contatos no Facebook. }\end{array}$ & \\
\hline & $\begin{array}{l}\text { BABeC } \\
\text { OM3 }\end{array}$ & $\begin{array}{l}\text { Quando estou extremamente satisfeito com um produto, } \\
\text { marca, serviço ou empresa, eu gosto de COMPARTILHAR } \\
\text { conteúdo positivo, relevante e/ou interessante com meus } \\
\text { contatos no Facebook.* }\end{array}$ & \\
\hline & $\begin{array}{l}\text { BABeP } \\
\text { UB1 }\end{array}$ & $\begin{array}{l}\text { Quando estou extremamente satisfeito com um produto, } \\
\text { marca, serviço ou empresa, eu gosto de PUBLICAR } \\
\text { conteúdo positivo, interessante e/ou relevante para meus } \\
\text { contatos no Facebook.* }\end{array}$ & \\
\hline & $\begin{array}{l}\text { BABeP } \\
\text { UB2 }\end{array}$ & $\begin{array}{l}\text { Em geral, gosto de PUBLICAR informações ou opiniões } \\
\text { positivas, interessantes e/ou relevantes sobre produtos, } \\
\text { marcas, serviços ou empresas de que gosto, admiro ou me } \\
\text { identifico. }\end{array}$ & \\
\hline & $\begin{array}{l}\text { BABeP } \\
\text { UB3 }\end{array}$ & $\begin{array}{l}\text { No Facebook, gosto de PUBLICAR conteúdo positivo, } \\
\text { interessante e/ou relevante sobre meus produtos, marcas ou } \\
\text { empresas favoritas. }\end{array}$ & \\
\hline
\end{tabular}

Nota. *itens não considerados nas escalas originalmente validadas e acrescentados pelos autores deste trabalho.

\section{Técnicas de análise}

As hipóteses deste estudo foram testadas por meio de modelos de equações estruturais (MEE). O presente trabalho utilizou o software WarpPLS 3.0 (Kock, 2012), uma ferramenta que permite a estimação de modelos de equações estruturais baseados em variância, estimados por Partial Least Squares (PLS) (Hair, Sarstedt, Ringle, \& Mena, 2011). Modelos de equações estruturais estimados por PLS têm sido usados frequentemente em alguns dos principais periódicos de marketing (Hair, Sarstedt 
et al., 2011). Vale ressaltar que, diferentemente dos modelos de equações estruturais baseados em covariância, os modelos de PLS não têm como objetivo reproduzir a matriz de covariância observada entre os indicadores (Lohmöller, 1989). Assim, não apresentam indicadores de ajuste agregados baseados na estatística quiquadrado, que testa a aderência da matriz de correlações reproduzidas à matriz observada.

As propriedades psicométricas do modelo de mensuração dos construtos propostos foram analisadas em termos de confiabilidade e validade convergente e discriminante. A extensão do efeito de variância comum ao método foi avaliada pelo teste de um fator de Harman (Podsakoff \& Organ, 1986). O possível efeito da variância comum ao método foi mitigado, pois uma das hipóteses testadas é de moderação e efeitos moderadores significantes não podem ser consequências de variância comum ao método (Siemsen, Roth, \& Oliveira, 2010).

As hipóteses do estudo foram testadas por meio da análise da significância estatística dos coeficientes estimados no modelo estrutural por bootstrapping (com 1.000 reamostragens) (Kock, 2012).

\section{Resultados}

\section{Análise descritiva da amostra}

Um total de 519 participantes completaram os questionários. Foram retirados 51 participantes que declararam não utilizar o Facebook. Do total de 468 questionários válidos, 358 participantes (76,5\%) declararam que já fizeram comunicação BAB-e positiva sobre produtos, serviços e marca na rede social. Estes 358 participantes compuseram a amostra final utilizada para testar as hipóteses do presente estudo (Figura 1). Destes, 280 (78\%) foram contatados por meio da lista de e-mails de alunos e ex-alunos de MBAs da FGV e 78 (22\%) a partir de redes sociais (Facebook e LinkedIn).

Dos 358 participantes que declararam que já fizeram comunicação BAB-e positiva sobre produtos, serviços e marcas na rede social, $53,1 \%$ são do sexo feminino. Em termos de estado civil, solteiros representaram 36,6\%; e casados, $52,0 \%$ da amostra. As faixas de idade predominantes foram de 25 a 34 anos $(43,3 \%)$ e de 35 a 44 anos $(34,4 \%)$. As faixas predominantes de renda familiar foram de $\mathrm{R} \$ 5.001,00$ a $\mathrm{R} \$ 10.000,00(29,1 \%)$ e mais de $\mathrm{R} \$ 10.000,00(55,9 \%)$. Em relação à frequência de acesso, 298 participantes $(83,2 \%)$ declararam acessar o site pelo menos uma vez ao dia, sendo que $71,5 \%$ dos participantes declararam acessar o site por diferentes meios, tais como celular ou tablet. Ao analisar a quantidade de contatos na lista de amigos, $74,3 \%$ dos participantes declararam possuir mais de 200 contatos.

\section{Modelo de mensuração}

As cargas fatoriais de todos os indicadores foram significantes $(\mathrm{p}<0,05)$ e superiores a 0,70 , exceto por BABeCUR1 e BABeCUR3, que tiveram cargas acima de 0,6. Os indicadores BS4 e RE2 tiveram carga abaixo de 0,5 e foram eliminados (Tabela 2 ). 
Tabela 2

Cargas Fatoriais e Indicadores de Confiabilidade do Modelo de Mensuração

\begin{tabular}{|c|c|c|c|c|c|}
\hline Construtos & Código do Indicador & Cargas & Composite Reliability & Alpha de Cronbach & AVE \\
\hline \multirow{3}{*}{$\begin{array}{l}\text { Preocupação com } \\
\text { outros } \\
\text { consumidores }\end{array}$} & POC1 & 0,871 & \multirow[t]{3}{*}{0,91} & \multirow[t]{3}{*}{0,852} & \multirow[t]{3}{*}{0,771} \\
\hline & $\mathrm{POC} 2$ & 0,881 & & & \\
\hline & POC3 & 0,882 & & & \\
\hline \multirow{3}{*}{$\begin{array}{l}\text { Desejo de ajudar a } \\
\text { empresa }\end{array}$} & DAE1 & 0,817 & \multirow[t]{3}{*}{0,89} & \multirow[t]{3}{*}{0,815} & \multirow[t]{3}{*}{0,73} \\
\hline & DAE2 & 0,873 & & & \\
\hline & DAE3 & 0,873 & & & \\
\hline \multirow[t]{3}{*}{ Benefício social } & BS1 & 0,830 & \multirow[t]{3}{*}{0,862} & \multirow[t]{3}{*}{0,759} & \multirow[t]{3}{*}{0,675} \\
\hline & BS2 & 0,836 & & & \\
\hline & BS3 & 0,799 & & & \\
\hline \multirow[t]{5}{*}{ Autoelevação } & AE1 & 0,835 & \multirow[t]{5}{*}{0,918} & \multirow[t]{5}{*}{0,888} & \multirow{5}{*}{0,693} \\
\hline & AE2 & 0,716 & & & \\
\hline & AE3 & 0,883 & & & \\
\hline & AE4 & 0,865 & & & \\
\hline & AE5 & 0,852 & & & \\
\hline \multirow{3}{*}{$\begin{array}{l}\text { Recompensas } \\
\text { econômicas }\end{array}$} & RE1 & 0,903 & \multirow[t]{3}{*}{0,932} & \multirow[t]{3}{*}{0,891} & \multirow[t]{3}{*}{0,822} \\
\hline & RE3 & 0,919 & & & \\
\hline & RE4 & 0,897 & & & \\
\hline \multirow{3}{*}{$\begin{array}{l}\text { Extravasar } \\
\text { emoções positivas }\end{array}$} & EEP1 & 0,832 & \multirow[t]{3}{*}{0,871} & \multirow[t]{3}{*}{0,778} & \multirow[t]{3}{*}{0,692} \\
\hline & EEP2 & 0,828 & & & \\
\hline & EEP3 & 0,835 & & & \\
\hline \multirow{3}{*}{$\begin{array}{l}\text { Forças dos laços } \\
\text { sociais }\end{array}$} & FLS1 & 0,746 & \multirow[t]{3}{*}{0,836} & \multirow[t]{3}{*}{0,705} & \multirow[t]{3}{*}{0,63} \\
\hline & FLS2 & 0,811 & & & \\
\hline & FLS3 & 0,822 & & & \\
\hline \multirow{9}{*}{$\begin{array}{l}\text { Boca a boca } \\
\text { eletrônica positiva }\end{array}$} & BABeCUR1 & 0,651 & \multirow[t]{9}{*}{0,934} & 0,92 & 0,614 \\
\hline & BABeCUR2 & 0,752 & & & \\
\hline & BABeCUR3 & 0,673 & & & \\
\hline & BABeCOM1 & 0,812 & & & \\
\hline & BABeCOM2 & 0,781 & & & \\
\hline & BABeCOM3 & 0,825 & & & \\
\hline & BABePUB1 & 0,826 & & & \\
\hline & BABePUB2 & 0,863 & & & \\
\hline & BABePUB3 & 0,839 & & & \\
\hline
\end{tabular}

A confiabilidade composta (Composite Reliability [CR]) e alpha de Cronbach de todos os construtos foram superiores a 0,7 (Tabela 2), indicando adequada confiabilidade do modelo de 
mensuração (Hair, Ringle, \& Sarstedt, 2011). Além disso, todos os construtos apresentaram AVE (Average Variance Extracted) superior a 0,5 (Hair, Ringle et al., 2011).

A raiz quadrada da variância média extraída (AVE) de cada construto (diagonal principal da Tabela 3) foi maior do que as correlações entre os construtos, o que sugere adequada validade discriminante (Fornell \& Larcker, 1981).

Tabela 3

Correlações entre os Construtos

\begin{tabular}{ccccccccc}
\hline Construtos & POC & DAE & BS & AE & RE & EEP & FLS & BABe \\
\hline POC & 0,878 & & & & & & & \\
DAE & 0,731 & 0,854 & & & & & & \\
BS & 0,527 & 0,495 & 0,822 & & & & & \\
AE & 0,451 & 0,432 & 0,712 & 0,832 & & & & \\
RE & 0,225 & 0,244 & 0,367 & 0,452 & 0,906 & & & \\
EEP & 0,687 & 0,667 & 0,693 & 0,627 & 0,265 & 0,832 & & \\
FLS & 0,174 & 0,118 & 0,149 & 0,101 & 0,117 & 0,118 & 0,794 & \\
BAB-e & 0,637 & 0,581 & 0,571 & 0,441 & 0,192 & 0,619 & 0,264 & 0,784 \\
\hline
\end{tabular}

Nota. Raiz quadrada da fração da variância extraída (AVE) é apresentada na diagonal principal.

Os valores de variance inflation factors (VIFs) observados foram inferiores a 5, indicando a ausência de problemas severos de multicolinearidade no modelo, como sugerido por Hair et al. (2009).

Finalmente, realizou-se o teste de um fator de Harman. Se a variância comum ao método é o principal determinante da variabilidade dos indicadores observados, uma análise fatorial exploratória deve extrair um único fator com autovalor superior a um ou o primeiro fator irá explicar a maior parte da variabilidade dos itens do questionário (Podsakoff \& Organ, 1986). O resultado da análise fatorial revelou sete distintos fatores, dessa maneira, explicando $66 \%$ da variância total dos itens, com o primeiro fator explicando 31,9\%. Embora não assegure a inexistência de variância comum ao método, esse teste indicou uma limitada presença deste problema.

Em suma, o modelo de mensuração passou por vários testes de validade convergente, validade discriminante, confiabilidade, multicolinearidade e variância comum ao método. Assim, os resultados indicaram que as propriedades psicométricas são adequadas.

\section{Modelo estrutural}

Três modelos foram estimados para testar as hipóteses previstas. Um primeiro modelo foi estimado incluindo somente variáveis de controle (Modelo 1). Posteriormente, foram acrescidos, respectivamente, nos Modelos 2 e 3, os efeitos principais e moderador para testar as hipóteses do estudo.

O R2 do modelo passou de 5\% (Modelo 1) para 54,3\% (Modelo 2), após a inclusão das variáveis independentes com efeito direto previsto na comunicação BAB-e positiva. Este aumento expressivo no poder de explicação do modelo indica que as variáveis elencadas nas hipóteses sobre efeitos diretos apresentam forte associação com o comportamento de boca a boca positivo (Tabela 4).

De acordo com os resultados, cinco das sete hipóteses apresentadas no estudo foram confirmadas (Tabela 4). Segundo o modelo 2, quanto maior a preocupação do indivíduo com outros consumidores (POC), mais intensa a comunicação BAB-e positiva no Facebook ( $b=0,288, p<0,01$ ), o que suporta H1. 
A hipótese $\mathrm{H} 2$, que previu um efeito positivo do desejo de ajudar a empresa (DAE) na comunicação boca a boca positiva, foi confirmada ( $b=0,147, \mathrm{p}<0,05)$.

A hipótese H3 também foi confirmada, revelando que a percepção do benefício social que a comunicação $\mathrm{BAB}$-e positiva traz ao indivíduo é outra importante motivação para o engajamento na comunicação BAB-e positiva $(b=0,235, p<0,01)$.

As hipóteses $\mathrm{H} 4$ e H5, que previram que autoelevação (AE) e recompensas econômicas (RE) afetariam positivamente a comunicação BAB-e positiva no Facebook, não foram confirmadas, uma vez que os coeficientes estimados não foram significantes.

Tabela 4

\section{Coeficientes do Modelo Estrutural}

\begin{tabular}{|c|c|c|c|}
\hline Variáveis Independentes & Modelo 1 & Modelo 2 & Modelo 3 \\
\hline \multicolumn{4}{|l|}{ Variáveis de Controle } \\
\hline Frequência Facebook & $0,149 * * *$ & 0,052 & $0,059 *$ \\
\hline Idade 15 a 24 & 0,037 & $-0,026$ & $-0,034$ \\
\hline Idade 25 a 34 & 0,116 & 0,058 & 0,049 \\
\hline Idade 35 a 44 & 0,129 & 0,073 & 0,066 \\
\hline Idade 45 a 54 & 0,126 & 0,078 & 0,081 \\
\hline Sexo Masculino & $-0,030$ & $-0,005$ & $-0,008$ \\
\hline Renda menor que 1500 & $-0,020$ & 0,018 & 0,027 \\
\hline Renda entre 1500 e 3000 & 0,013 & 0,022 & 0,013 \\
\hline Renda entre 3000 e 5000 & $0,140 * * *$ & $0,097 * *$ & $0,099^{* *}$ \\
\hline Renda entre 5000 e 10000 & 0,005 & 0,013 & 0,016 \\
\hline \multicolumn{4}{|l|}{ Efeitos diretos } \\
\hline POC & & $0,288 * * *$ & $0,314 * * *$ \\
\hline DAE & & $0,147 * * *$ & $0,122 * *$ \\
\hline BS & & $0,235^{* * *}$ & $0,221 * * *$ \\
\hline $\mathrm{AE}$ & & $-0,018$ & $-0,023$ \\
\hline $\mathrm{RE}$ & & $-0,043$ & $-0,028$ \\
\hline EEP & & $0,161 * * *$ & $0,173^{* * *}$ \\
\hline FLS & & $0,123 * * *$ & $0,126^{* * *}$ \\
\hline \multicolumn{4}{|l|}{ Efeito moderador } \\
\hline FLS*POC & & & $0,103^{* * *}$ \\
\hline $\mathrm{R}^{2}$ & 0,050 & $0,543 * * *$ & $0,552 * * *$ \\
\hline Average VIF & 3,103 & 2,805 & 2,724 \\
\hline
\end{tabular}

Nota. significante a $* 10 \%$; $* * 5 \%$ e $* * * 1 \%$.

A hipótese H6, que previu que o desejo de extravasar emoções positivas (EEP) afeta positivamente a comunicação BAB-e positiva, foi confirmada $(b=0,161, p<0,01)$. Por fim, as hipóteses $\mathrm{H} 7 \mathrm{a}$ e H7b também foram confirmadas. A força dos laços sociais (FLS) está positivamente associada à comunicação BAB-e positiva $(b=0,126$ e $p<0,01)$ e, além disso, quanto maior a percepção do indivíduo das forças dos laços sociais (FLS) que compõem sua rede de contatos Facebook, maior é o efeito da 
preocupação com outros consumidores (POC) na comunicação $\mathrm{BAB}$-e positiva (b=0,103 e p<0,01, Modelo 3).

Testou-se, também, se os resultados são semelhantes para as diferentes fontes de captação dos respondentes (alunos de MBAs da FGV ou contatos no Facebook ou LinkedIn). Uma análise multigrupo indicou que as diferenças entre os coeficientes estruturais não foram significantes, o que sugere que os resultados são semelhantes para respondentes de ambas as fontes.

\section{Conclusões}

O Facebook, maior site de redes sociais do Brasil, com 67 milhões de usuários ativos no final de 2012 (Facebook, 2012), facilitou a disseminação da comunicação BAB-e positiva entre consumidores através da internet. A consolidação do site como uma plataforma de acesso móvel aumentou o seu potencial de compartilhamento de experiências de consumo em tempo real, através de diferentes dispositivos (smartphones e tablets) e formatos (texto, fotos e vídeos). No total, quase $65 \%$ da base de usuários acessaram o serviço por me io de dispositivos móveis em 2012 (Facebook, 2012).

Ao analisar as motivações subjacentes ao comportamento de comunicação BAB-e positiva no site a partir da realidade do mercado brasileiro, o estudo corrobora, em parte, resultados de pesquisas anteriores realizadas em outros países (Hennig-Thurau et al., 2004; Jeong \& Jang, 2011; Yoo et al., 2013). Os resultados sugerem quatro significantes motivações distintas de natureza social e psicológica: (a) preocupação com outros consumidores; (b) benefício social; (c) desejo de compartilhar emoções positivas; (e) desejo de ajudar a empresa.

O estudo indica, também, que o altruísmo desempenha um papel importante como motivador da comunicação BAB-e positiva no Facebook entre os consumidores brasileiros. Este achado suporta o estudo de Yoo, Sanders e Moon (2013), que, ao analisar a comunicação BAB-e no contexto de shopping virtual de compras na Coreia, sugere que as motivações intrínsecas (por exemplo, desejo de ajudar outros consumidores) são mais importantes do que as motivações extrínsecas, tais como recompensas econômicas.

O estudo aponta, ainda, forte relação entre a percepção do benefício social que a comunicação BAB-e positiva traz ao indivíduo e o comportamento de comunicação BAB-e positiva. Ao considerar que o principal propósito do Facebook é justamente permitir a socialização entre amigos, colegas e outras pessoas, a comunicação BAB-e positiva de produtos e marcas surge como uma forma de facilitar a integração e o estabelecimento de novos contatos na rede social. Benefícios sociais também foram identificados como a mais importante motivação da comunicação BAB-e nos estudos de Hennig-Thurau et al. (2004) e Yoo et al. (2013).

Em sentido contrário aos resultados do estudo de Hennig-Thurau et al. (2004), as hipóteses que previram que a autoelevação e as recompensas econômicas afetam positivamente a comunicação BABe positiva não foram confirmadas. Uma possível explicação para tal não confirmação pode residir no fornecimento de respostas consideradas socialmente desejáveis, mais esperadas e aceitas pela sociedade, em função do desejo de aceitação social, pois os respondentes afirmaram não ser motivados por incentivos instrumentais e de natureza mais egoísta relacionadas à obtenção de ganhos financeiros e status a partir das relações interpessoais do Facebook. O presente estudo investigou a comunicação BAB-e no Facebook, um ambiente de socialização, diversão, compartilhamento de experiências pessoais e fornecimento de ajuda e suporte, no qual as pessoas geralmente utilizam suas identidades verdadeiras e relacionam-se com amigos, familiares e colegas de trabalho. É razoável, portanto, esperar que, neste ambiente, exista uma tensão e potencial conflito entre as relações de amizade e de negócios. Isso porque o indivíduo que adere a campanhas promocionais para obter ganhos financeiros ou se autopromova através da divulgação e compartilhamento de informações favoráveis sobre produtos e marcas para sua rede de contatos, com o intuito de elevar seu status, pode ser acusado, pelos demais 
membros, de minar o ideal de partilha e cuidado existente no grupo, conforme abordado nos estudos de Grayson (2007), Kozinets et al. (2010) e Pereira (2010).

O teste empírico deu suporte ao papel moderador da força dos laços sociais na relação entre preocupação com outros consumidores e a comunicação BAB-e positiva, bem como identificou uma associação positiva entre a força dos laços sociais e a comunicação BAB-e positiva. Desta forma, confirmou o papel-chave da teoria da força dos laços fracos de Granovetter (1973) para o entendimento deste comportamento.

Como contribuição teórica, este trabalho acrescenta o papel da força dos laços sociais como efeito moderador da relação entre motivações e comunicação BAB-e, que havia sido tratado anteriormente apenas em seu efeito direto. Permite, também, uma expansão do alcance da teoria sobre motivadores do comportamento de comunicação BAB-e no mercado brasileiro, haja vista que poucos estudos desenvolvidos sobre motivações da comunicação $\mathrm{BAB}$-e foram realizados em países outros que o Brasil e estudos anteriores mostraram que estas motivações têm seus efeitos alterados em função do ambiente nacional. Os resultados empíricos confirmaram a relevância dos fatores motivacionais e da variável moderadora do modelo teórico proposto para o entendimento do fenômeno. $\mathrm{O}$ estudo ampliou o modelo de motivações de Hennig-Thurau et al. (2004) ao incluir o construto força dos laços sociais e demonstrar que a variável afeta positivamente a comunicação $\mathrm{BAB}$-e positiva, bem como modera a relação entre a preocupação com outros consumidores e a comunicação BAB-e positiva.

Por sua vez, em termos gerenciais, o estudo pode contribuir para uma discussão de como os profissionais de marketing das empresas podem trabalhar para explorar o potencial da comunicação BAB-e no Facebook. Primeiro, o estudo sugere que o comportamento de comunicação BAB-e positiva é amplamente praticado entre seus usuários no Brasil. Através de funcionalidades específicas, os consumidores curtem, compartilham e publicam conteúdo positivo sobre produtos, serviços e marcas para a rede de contatos de forma fácil e rápida. Portanto, as empresas devem considerar o Facebook em suas estratégias de marketing e comunicação. Além disso, os gestores devem monitorar com frequência o conteúdo divulgado sobre suas empresas e estar aptos para estabelecer um verdadeiro diálogo com seus consumidores através do site. Segundo, os resultados fornecem uma visão das motivações que levam os consumidores brasileiros a emitirem comunicação BAB-e positiva no Facebook. Conforme revelado no estudo, elas são de natureza social e psicológica, e não puramente econômica. A partir deste entendimento, os profissionais de marketing podem desenvolver ações mais efetivas para estimular o comportamento. Por exemplo, tendo em vista o impacto relevante do desejo por interações sociais na comunicação BAB-e positiva, as empresas devem criar conteúdo com alto potencial de conversação que ajude os usuários a relacionarem-se uns com os outros.

As empresas também devem identificar e encorajar os líderes de opinião e compradores atuais a propagarem informações positivas sobre os produtos, serviços e marcas para sua rede de contatos na rede social. Nesse sentido, as empresas podem disponibilizar a opção Curtir em diversos lugares da internet onde sua marca e produtos estão presentes, facilitando, desta forma, o processo de recomendação por parte dos consumidores.

O estudo apresenta limitações que podem ser abordadas em pesquisas futuras. Primeira, a amostra utilizada é não probabilística, o que restringe a validade externa dos resultados. Foi possível observar, por exemplo, que a amostra sobrerrepresenta respondentes com nível de renda mais elevado. Neste sentido, pesquisas futuras podem proceder a um levantamento de dados por meio de amostragem probabilística.

Segunda, não é possível estabelecer claras relações causais entre as variáveis dado que o desenho não experimental não permite excluir problemas de endogeneidade. Estudos futuros podem lançar mão de desenhos de pesquisa experimentais que permitam inferência causal com maior validade interna.

Terceira, o comportamento foi baseado em fatos acontecidos no passado, após a sua ocorrência, não havendo controle sobre as variáveis em estudo e tendo de apoiar-se em informações derivadas da memória dos respondentes. Além disso, conforme abordado neste artigo, grande parte das variáveis 
foram medidas com base na percepção dos respondentes, o que pode afetar os resultados em função do fornecimento de respostas consideradas politicamente corretas. Por conseguinte, as hipóteses não confirmadas, relacionadas à obtenção de recompensas econômicas e de autoelevação a partir das relações interpessoais do Facebook, merecem ser aprofundadas em pesquisas futuras. Sugere-se que novas pesquisas também utilizem metodologias qualitativas, como, por exemplo, a netnografia, técnica que permite a inserção do pesquisador no dia a dia do grupo virtual investigado.

Quarta, um efeito de social desirability pode ter afetado os resultados. Por exemplo, a não significância das recompensas financeiras como motivadoras do BAB-e pode ser explicada por essa razão. Sugere-se que estudos futuros incluam uma escala de social desirability (Crowne \& Marlowe, 1960) de maneira a controlar esta hipótese alternativa.

Como forma de ampliar o entendimento do fenômeno, o efeito moderador de novas variáveis na relação dos fatores motivadores e a comunicação boca a boca positiva pode ser testado em estudos futuros. Assim, poderiam ser incluídas variáveis relacionadas a traços de personalidade e relacionamento interpessoal, como, por exemplo, narcisismo e confiança.

Por fim, não constituiu objeto do presente estudo a comunicação BAB-e negativa. Entender sua dinâmica nas redes sociais, contemplando suas motivações e impacto no processo de compra do consumidor e na reputação das empresas, é de extrema relevância para os profissionais de comunicação e marketing.

\section{Referências}

Antonakis, J., Bendahan, S., Jacquart, P., \& Lalive, R. (2010). On making causal claims: a review and recommendations. The Leadership Quarterly, 21(6), 1086-1120. doi: 10.1016/j.leaqua.2010.10.010

Arndt, J. (1967). Role of product-related conversations in the diffusion of a new product. Journal of Marketing Research, 4(3), 291-295. doi: 10.2307/3149462

Awad, N. F., \& Ragowsky, A. (2008). Establishing trust in electronic commerce through online word of mouth: an examination across genders. Journal of Management Information Systems, 24(4), 101-121. doi: 10.2753/MIS0742-1222240404

Balasubramanian, S., \& Mahajan, V. (2001). The economic leverage of the virtual community. Int. J. Electron. Commerce, 5(3), 103-138.

Botelho, D., \& Almeida, A. (2009). Construção de questionários. In D. Botelho \& D. Zouain (Eds.), Pesquisa quantitativa em administração (pp. 90-108). São Paulo: Atlas.

Boyd, D. M., \& Ellison, N. B. (2007). Social network sites: definition, history, and scholarship. Journal of Computer-Mediated Communication, 13(1), 210-230. doi: 10.1111/j.1083-6101.2007.00393.x

Breazeale, M. (2009). Word of mouse an assessment of electronic word-of-mouth research. International Journal of Market Research, 51(3), 297-318. doi: 10.2501/S1470785309200566

Bronner, F., \& Hoog, R. (2010). Vacationers and eWOM: who posts, and why, where, and what? Journal of Travel Research, 50(1), 15-26. doi: 10.1177/004728750935532

Brown, J. J., \& Reingen, P. H. (1987). Social ties and word-of-mouth referral behavior. Journal of Consumer Research, 14(3), 350-362. doi: 10.2307/2489496 
Brown, T. J., Barry, T. E., Dacin, P. A., \& Gunst, R. F. (2005). Spreading the word: investigating antecedents of consumers' positive word-of-mouth intentions and behaviors in a retailing context. Journal of the Academy of Marketing Science, 33(2), 123-138. doi: 10.1177/0092070304268417

Buttle, F. A. (1998). Word of mouth: understanding and managing referral marketing. Journal of Strategic Marketing, 6(3), 241-254. doi: 10.1080/096525498346658

Chevalier, J. A., \& Mayzlin, D. (2006). The effect of word of mouth on sales: online book reviews. Journal of Marketing Research, 43(3), 345-354. doi: 10.1509/jmkr.43.3.345

Chu, S.-C., \& Choi, S. M. (2011). Electronic word-of-mouth in social networking sites: a cross-cultural study of the United States and China. Journal of Global Marketing, 24(3), 263-281. doi: 10.1080/08911762.2011.592461

Chu, S.-C., \& Kim, Y. (2011). Determinants of consumer engagement in electronic word-of-mouth (eWOM) in social networking sites. International Journal of Advertising, 30(1), 47-75. doi: 10.2501/IJA-30-1-047-075

Crowne, D. P., \& Marlowe, D. (1960). A new scale of social desirability independent of psychopathology. Journal of Consulting Psychology, 24(4), 349-354. doi: 10.1037/h0047358

Dellarocas, C. (2003). The digitization of word of mouth: promise and challenges of online feedback mechanisms. Management Science, 49(10), 1407-1424. doi: 10.1287/mnsc.49.10.1407.17308

Dichter, E. (1966). How word-of-mouth advertising works. Harvard Business Review, 44(6), 147-160.

Duan, W., Gu, B., \& Whinston, A. (2008). The dynamics of online word-of-mouth and product sales: an empirical investigation of the movie industry. Journal of Retailing, 84(2), 233-242. doi: 10.1016/j.jretai.2008.04.005

Engel, J. F., Blackwell, R. D., \& Miniard, P. W. (1993). Consumer behaviour. International edition (8th ed.). Fort Worth: Dryden Press.

Facebook. (2012). Annual report - 2012. Menlo Park, CA: Autor. Recuperado de http://files.shareholder.com/downloads/AMDA-NJ5DZ/3655378373x0x658233/46826077D2FD-4E84-9BBE-C3F844B547A0/FB_2012_10K.pdf

Fornell, C., \& Larcker, D. F. (1981). Evaluating structural equation models with unobservable variables and measurement error. Journal of Marketing Research, 18(1), 39-50. doi: 10.2307/3151312

Fowler, F. J. (2014). Survey research methods (5th ed.). Boston, MA: Sage Publications.

Goyette, I., Ricard, L., Bergeron, J., \& Marticotte, F. (2010). e-WOM scale: word-of-mouth measurement scale for e-services context. Canadian Journal of Administrative Sciences / Revue Canadienne Des Sciences de l'Administration, 27(1), 5-23. doi: 10.1002/cjas.129

Granovetter, M. S. (1973). The strength of weak ties. American Journal of Sociology, 78(6), 1360-1380. doi: $10.2307 / 2776392$

Grayson, K. (2007). Friendship versus business in marketing relationships. Journal of Marketing, 71(4), 121-139. doi: 10.1509/jmkg.71.4.121

Groeger, L., \& Buttle, F. (2014). Word-of-mouth marketing influence on offline and online communications: evidence from case study research. Journal of Marketing Communications, 20(1/2), 21-41. doi: 10.1080/13527266.2013.797736

Hair, J., Black, W., Babin, B., \& Anderson, R. (2009). Multivariate data analysis (7th ed.). Upper Saddle River, NJ: Prentice Hall. 
Hair, J., Ringle, C. M., \& Sarstedt, M. (2011). PLS-SEM: indeed a silver bullet. The Journal of Marketing Theory and Practice, 19(2), 139-152. doi: 10.2753/MTP1069-6679190202

Hair, J., Sarstedt, M., Ringle, C., \& Mena, J. (2011). An assessment of the use of partial least squares structural equation modeling in marketing research. Journal of the Academy of Marketing Science, 40(3), 414-433. doi: 10.1007/s11747-011-0261-6

Hennig-Thurau, T., Gwinner, K. P., Walsh, G., \& Gremler, D. D. (2004). Electronic word-of-mouth via consumer-opinion platforms: what motivates consumers to articulate themselves on the Internet? Journal of Interactive Marketing, 18(1), 38-52. doi: 10.1002/dir.10073

Jeong, E., \& Jang, S. (2011). Restaurant experiences triggering positive electronic word-of-mouth (eWOM) motivations. International Journal of Hospitality Management, 30(2), 356-366. doi: 10.1016/j.ijhm.2010.08.005

Kock, N. (2012). WarpPLS 3.0 user manual. Texas: ScriptWarp Systems.

Kozinets, R. V., Valck, K. de, Wojnicki, A. C., \& Wilner, S. J. S. (2010). Networked narratives: understanding word-of-mouth marketing in online communities. Journal of Marketing, 74(2), 7189. doi: $10.1509 / j \mathrm{jkg} .74 .2 .71$

Lee, H., Lee, D., Il, Kim, T., \& Lee, J. (2013). The moderating role of socio-semantic networks on online buzz diffusion. Journal of Business Research, 66(9), 1367-1374. doi: 10.1016/j.jbusres.2012.02.038

Lee, M., \& Youn, S. (2009). Electronic word of mouth (eWOM) How eWOM platforms influence consumer product judgement. International Journal of Advertising, 28(3), 473-499. doi: $10.2501 / \mathrm{S} 0265048709200709$

Lohmöller, J. B. (1989). Latent variable path modeling with partial least squares. Physica-Verlag Heidelberg.

Oliver, R. L. (1980). A cognitive model of the antecedents and consequences of satisfaction decisions. Journal of Marketing Research, 17(4), 460-469. doi: 10.2307/3150499

Pereira, M. (2010, setembro). Amor ou interesse? Novas construções para a recomendação boca a boca. Anais do Encontro Nacional da Associação Nacional de Pós-Graduação e Pesquisa em Administração, Rio de Janeiro, RJ, Brasil, 34.

Podsakoff, P. M., MacKenzie, S. B., \& Podsakoff, N. P. (2012). Sources of method bias in social science research and recommendations on how to control it. Annual Review of Psychology, 63, 539-569. doi: 10.1146/annurev-psych-120710-100452

Podsakoff, P. M., \& Organ, D. W. (1986). Self-reports in organizational research: problems and prospects. Journal of Management, 12(4), 531-544. doi: 10.1177/014920638601200408

Price, L. L., Feick, L. F., \& Guskey, A. (1995). Everyday market helping behavior. Journal of Public Policy \& Marketing, 14(2), 255-266.

Siemsen, E., Roth, A., \& Oliveira, P. (2010). Common method bias in regression models with linear, quadratic, and interaction effects. Organizational Research Methods, 13(3), 456-476. doi: $10.1177 / 1094428109351241$

Sun, T., Youn, S., Wu, G., \& Kuntaraporn, M. (2006). Online word-of-mouth (or mouse): an exploration of its antecedents and consequences. Journal of Computer-Mediated Communication, 11(4), 1104-1127. doi: 10.1111/j.1083-6101.2006.00310.x 
Sundaram, D., Mitra, K., \& Webster, C. (1998). Word-of-mouth communications: a motivational analysis. Advances in Consumer Research, 25(1), 527-531.

Swan, J. E., \& Oliver, R. L. (1989). Postpurchase communications by consumers. Journal of Retailing, $65(4), 516-533$.

Sweeney, J., Soutar, G., \& Mazzarol, T. (2013). Factors enhancing word-of-mouth influence: positive and negative service-related messages. European Journal of Marketing, 48(1), 336-359. doi: 10.1108/EJM-06-2012-0336

Vargo, S. L., \& Lusch, R. F. (2004). Evolving to a new dominant logic for marketing. Journal of Marketing, 68(1), 1-17. doi: 10.1509/jmkg.68.1.1.24036

Wolny, J., \& Mueller, C. (2013). Analysis of fashion consumers' motives to engage in electronic wordof-mouth communication through social media platforms. Journal of Marketing Management, 29(5/6), 562-583. doi: 10.1080/0267257X.2013.778324

Yoo, C. W., Sanders, G. L., \& Moon, J. (2013). Exploring the effect of e-WOM participation on eloyalty in e-commerce. Decision Support Systems, 55(3), 669-678. doi: 10.1016/j.dss.2013.02.001

\section{Dados dos Autores}

Daniel Buarque Tubenchlak

Praia de Botafogo, 190, 22250-900, Rio de Janeiro, RJ, Brasil. E-mail: daniel.buarque@hotmail.com

Diego de Faveri

Praia de Botafogo, 190, 22250-900, Rio de Janeiro, RJ, Brasil. E-mail: diego.lima@fgv.br

Marco Tulio Zanini

Praia de Botafogo, 190, 22250-900, Rio de Janeiro, RJ, Brasil. E-mail: marco.zanini@ fgv.br

Rafael Goldszmidt

Praia de Botafogo, 190, 22250-900, Rio de Janeiro, RJ, Brasil. E-mail: rafael.goldszmidt@fgv.br 